\title{
Depinning and Collective Dynamics of Magnetically Driven Colloidal Monolayers
}

\author{
Pietro Tierno* \\ Estructura i Constituents de la Matèria, Universitat de Barcelona, Avinguda Diagonal 647, 08028 Barcelona, Spain \\ and Institut de Nanociència i Nanotecnologia IN ${ }^{2}$ UB, Universitat de Barcelona, 08028 Barcelona, Spain
}

(Received 20 August 2012; published 9 November 2012)

\begin{abstract}
We study the collective dynamics of interacting paramagnetic colloids transported via a magnetic ratchet effect above a modulated periodic potential. Upon increasing the modulation frequency, the particles undergo a series of dynamic transitions, from a continuous smectic flow to a disorder flow, and later enter into a two phase flow regime, ending in a complete pinned state. In the disordered phase, the system organizes into density waves due to traffic jams, as in granular systems, while the two phase flow regime shows strong similarities with plastic flow in vortex matter. Finally, it is shown that induced attractive interactions between the moving colloids lead to enhancement of the particle current due to formation of condensed chains traveling along the modulated landscape.
\end{abstract}

DOI: 10.1103/PhysRevLett.109.198304

PACS numbers: 82.70.Dd, 05.40.Fb, 05.60.Cd

The pinning-depinning transition in systems driven above a corrugated potential is a rich and complex phenomenon, underlying the physics of many condensed matter systems. Examples are numerous and include, among others, vortices in type II superconductors [1], charge density waves [2], dislocations [3], elastic strings [4], magnetic bubbles [5], sliding Wigner crystals [6], and driven Frenkel-Kontorova systems [7]. The emerging physics encompassing all such systems is a nonequilibrium phase transition from a pinned to a moving state upon reaching a critical threshold force. Recent experiments with random [8] or periodic [9] potentials, have shown that colloidal particles represent an experimentally accessible model system where dynamical phases in pinning potentials can be studied in real time and space and with single particle resolution. In particular, when driven by an electric field above a random pinning potential, charged colloids show elastic or plastic depinning, in the presence of weak or strong disorder strength [8]. On the other hand, when a colloidal crystal is translated through an optical lattice, friction induces mismatch dislocations between the particles and the sliding potential, with propagation of excitations (kinks or antikinks) along the force direction [10].

The experimental scenarios developed until now, however, focused mainly on the dynamics and depinning of colloids driven through potential landscapes by increasing the applied force. In contrast, this Letter experimentally investigates the opposite situation, i.e., the phase behavior and dynamics of interacting particles when they transit from a continuous sliding state to a completely pinned one. In particular, a deterministic (athermal) ratchet effect induces synchronized directed motion of paramagnetic colloids above a frequency modulated magnetic landscape. Increasing the frequency, the particles desynchronize with the ratchet potential, decreasing and randomizing their speed, resulting in local traffic jams and density waves on a large scale. At higher frequencies, the jams convert into pinned domains surrounded by flowing colloidal rivers. Finally, we show a way to enhance the particle current in situ, inducing attractive dipolar forces between the moving colloids.

The colloids used in the experiments consisted of aqueous suspensions of monodisperse (CV 1.9\%) paramagnetic particles with diameter $d=1.05 \mu \mathrm{m}$, and magnetic volume susceptibility $\chi=1.1$ (Dynabeads MyOne, Dynal). The particles were deposited on top of the periodic potential generated by a uniaxial ferrite garnet film (FGF), grown by dipping liquid phase epitaxy [11]. The FGF was characterized by ferromagnetic domains having alternating magnetization with periodicity $\lambda=2.6 \mu \mathrm{m}$, and separated by Block Walls (BWs), i.e., narrow transition regions where the magnetic stray field of the film is maximal [see Fig. 1(a)]. After sedimentation, the particles were attracted by the BWs, forming parallel chains, spaced $\lambda / 2$, and with exclude mutual passage [see Fig. 1(b)]. In contrast to previous works [12], the ratchet effect was realized by applying an external rotating magnetic field in the $(y, z)$ plane, $\left.\boldsymbol{H} \equiv\left[H_{y} \cos (\omega t) \boldsymbol{e}_{y}+H_{z} \sin (\omega t) \boldsymbol{e}_{z}\right)\right]$, with frequency $\omega$, and amplitude $H_{0}=\sqrt{H_{y}^{2}+H_{z}^{2}}$ (more details are given in the Supplemental Material [13]). In most of the experiments, we fixed the amplitude of the field at $H_{0}=$ $900 \mathrm{~A} / \mathrm{m}$ with $H_{y}=H_{z}$, and varied the frequency and the area density $\rho=N / A$, with $N$ being the number of particles and $A=140 \times 105 \mu \mathrm{m}^{2}$ the observation area. The sliding dynamics at high frequency was induced by decreasing the magnetic attraction of the film. In particular, the FGF had a smaller $\lambda$ compared to Ref. [12], and was coated with a $1 \mu \mathrm{m}$ thick layer of a photoresist (AZ1512, Microchemicals) by using standard spin coating and UV photocrosslinking [14]. At the particle elevation, the magnetic potential was $4 k_{B} T\left(10 k_{B} T\right.$ with no 

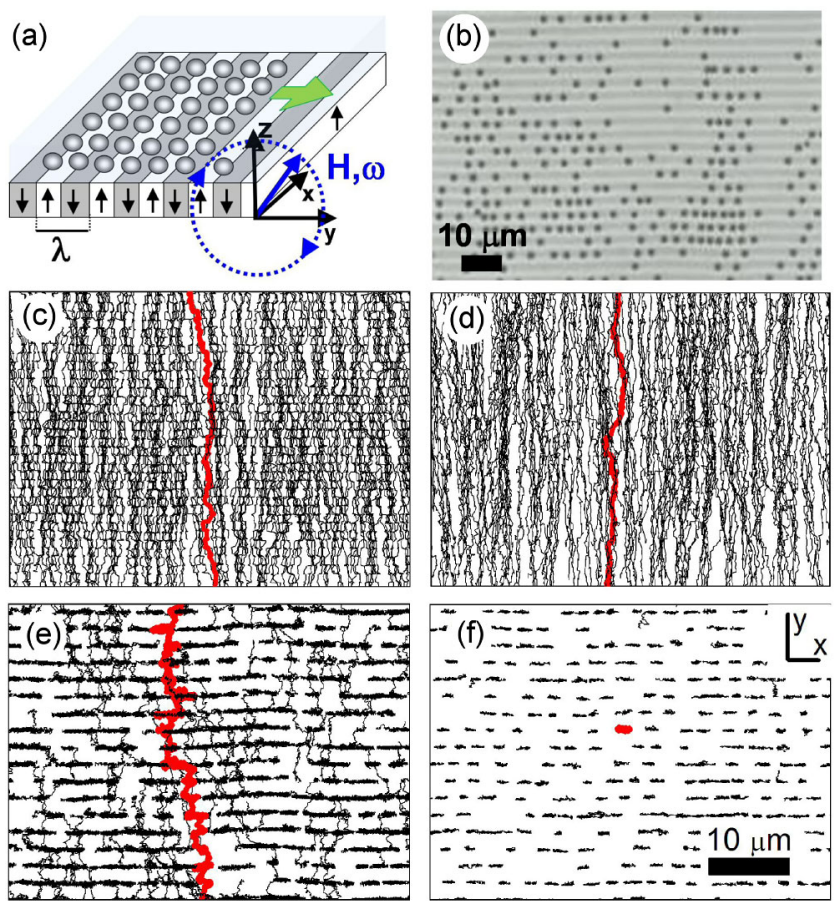

FIG. 1 (color online). (a) Schematic showing the paramagnetic colloids above a FGF with spatial periodicity $\lambda=2.6 \mu \mathrm{m}$ and subjected to an external magnetic field $H$ rotating in the $(y, z)$ plane. (b) Microscope image showing a diluted sample of particles (areal density $\rho=0.03 \mu \mathrm{m}^{-2}$ ) and the magnetic pattern. Images (c)-(f) show experimental trajectories of the particles [one highlighted in gray (red), $\rho=0.22 \mu \mathrm{m}^{-2}$ ] in the various regimes of motion with $H_{0}=900 \mathrm{~A} / \mathrm{m}$ and $\omega=$ $19 \mathrm{~s}^{-1}$ (c), $251 \mathrm{~s}^{-1}$ (d), $484 \mathrm{~s}^{-1}$ (e), and $628 \mathrm{~s}^{-1}$ (f). For clarity, the data show a small overview $\left(44 \times 44 \mu \mathrm{m}^{2}\right)$ of the whole observation area $\left(140 \times 105 \mu \mathrm{m}^{2}\right)$. Corresponding movies can be found in the Supplemental Material [13].

coating), as determined by statistical analysis on dilute samples $[15,16]$.

Figures 1(c)-1(f) show typical particle trajectories $\left(x_{i}(t), y_{i}(t)\right)$ for different frequencies, illustrating the four regimes observed: (i) a locked smectic flow, (ii) a disordered flow, (iii) a two phase flow, and (iv) a pinned state. More quantitative characterizations can be found in Fig. 2, which shows the average velocity along the direction of motion, $\left\langle v_{y}(t)\right\rangle=\left\langle\frac{1}{N} \sum_{i} \frac{d y_{i}}{d t}\right\rangle$ [Fig. 2(a)], the fraction of sixfold coordinated particles, $\left\langle P_{6}\right\rangle=\left\langle\frac{1}{N} \sum_{i} \delta\left(z_{i}-6\right)\right\rangle$ [Fig. 2(b)], the fraction $\phi_{s}\left(\phi_{0}\right)$ of colloids moving with the same velocity (with zero velocity) [Fig. 2(c)], and the transversal diffusion coefficient of the particles, $D_{x}=$ $\left\langle\left[x_{i}(t)-x_{i}(0)\right]^{2}\right\rangle / 2 t\left[\right.$ Fig. 2(d)]. Here $z_{i}$ is the coordination number of each particle obtained from a Voronoi tessellation.

In regime I, which occurs for $\omega \leq 110 \mathrm{~s}^{-1}$, all particles are perfectly synchronized with the ratchet potential, moving with a constant maximum speed given by $v=\lambda \omega / 2 \pi$, with $\phi_{0}=0, \phi_{s}=1$. The colloidal monolayer displays smectic order with dislocations, and this type of ordering is
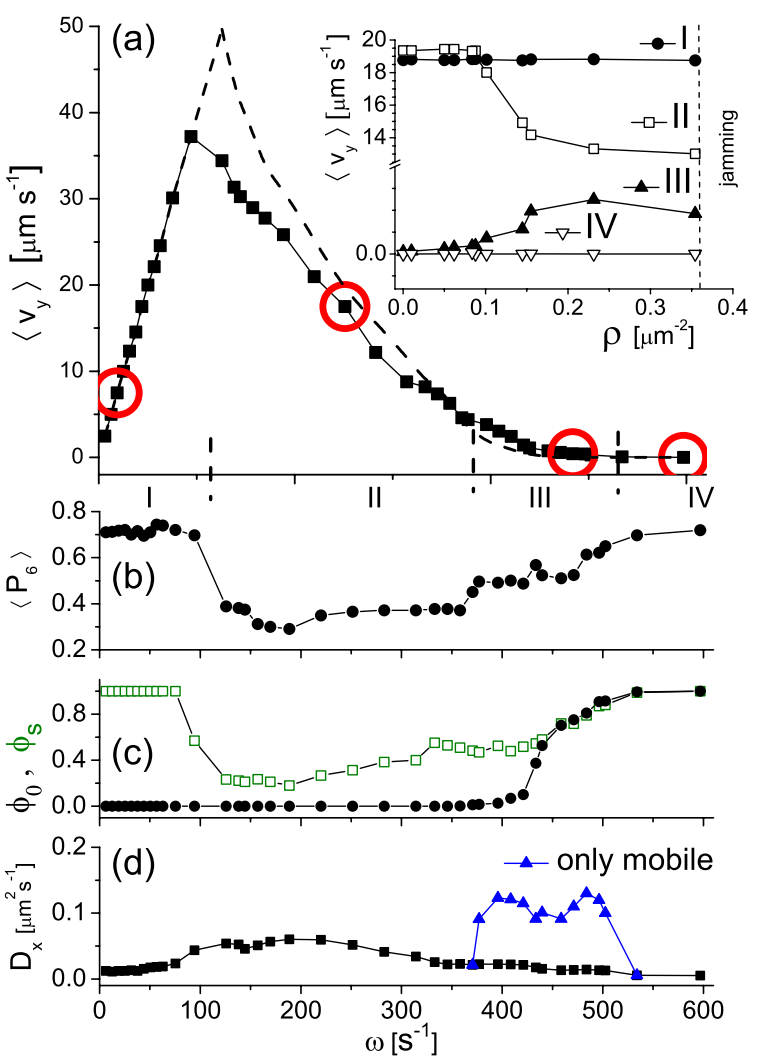

FIG. 2 (color online). (a) Average velocity $\left\langle v_{y}\right\rangle$ in the driving direction $(y)$ versus $\omega$ for a single particle (dashed line) and a collection of particles with density $\rho=0.22 \mu \mathrm{m}^{-2}$ (black squares). Gray (red) circles denote the location of the trajectories shown in Figs. 1(c)-1(f). Inset shows $\left\langle v_{y}\right\rangle$ versus $\rho$ in the four regimes, with frequencies corresponding to Figs. 1(c)-1(f). (b) Average fraction of sixfold coordinated particles $\left\langle P_{6}\right\rangle$ versus $\omega$. (c) Gray (green) empty squares denote the fraction of particles having the same velocity $\phi_{s}$, black points denote the fraction of particles having zero velocity $\phi_{0}$, both versus $\omega$. (d) Transversal diffusion coefficient $D_{x}$ versus frequency. Gray (blue) triangles indicate the values of $D_{x}$ for the mobile colloids in regime III.

due to the periodicity of the magnetic substrate. As a consequence, the fraction of sixfold coordinated particles is $P_{6} \sim 0.7$, i.e., less than one as for a triangular crystal, and similar to the value predicted in simulations of particles pinned by a one-dimensional potential [17]. In the transverse direction, the moving colloids show small Gaussian fluctuations, with a diffusion coefficient $D_{x}=$ $0.06 \mu \mathrm{m}^{2} \mathrm{~s}^{-1}$ for $\omega=19 \mathrm{~s}^{-1}$. In regime II, $110 \mathrm{~s}^{-1}<$ $\omega \leq 375 \mathrm{~s}^{-1}$, the overdamped particles start to desynchronize with the magnetic potential, decreasing the average speed of the system. The system becomes highly disordered with $P_{6} \sim 0.4$ and $\phi_{s} \sim 0.4$. In this situation, $D_{x}=0.05 \mu \mathrm{m}^{2} \mathrm{~s}^{-1}$ for $\omega=251 \mathrm{~s}^{-1}$, thus almost unchanged from regime $\mathrm{I}$, since faster particles do not surpass the slower ones, but rather join them creating traffic jams along the direction of motion (see Video 2 in the Supplemental Material [13]). In regime III, 
$375 \mathrm{~s}^{-1}<\omega \leq 520 \mathrm{~s}^{-1}$, some particles become pinned above the BWs, creating small domains randomly located on the film. The size of these domains is not fixed, but changes with time as the colloidal monolayer flows [18]. The average speed of the system decreases faster than in regime II, while the spatial order starts to slowly increase; $P_{6}>0.5$ since the pinned particles align with the BWs, restoring the spatial periodicity of the film. Increasing the initial density $\rho$, the size of the pinned domains increases and above a threshold value $\rho=0.36 \mu \mathrm{m}^{2}$ the domains percolate, jamming the whole system. For lower $\rho$, however, the mobile particles are forced by the pinned domains to undertake tortuous trajectories between them [see Fig. 1(e)]. As a consequence, the transversal diffusion coefficient of the mobile particles increases 1 order of magnitude, $D_{x}=0.13 \mu \mathrm{m}^{2} \mathrm{~s}^{-1}$ for $\omega=484 \mathrm{~s}^{-1}$. This regime resembles, to a large extent, the plastic-flow regime observed for vortices driven through type II superconductors in the presence of strong quenched disorder. Here however, the disorder is dynamic, and caused by the continuous interactions with the surrounding particle bath. Finally, for $\omega>520 \mathrm{~s}^{-1}$ all particles became pinned, $\phi_{0}=\phi_{s}=1$, and the smectic order completely reenters, with $P_{6}=0.7$.

The complex phases observed in regimes II and III are the result of collective effects, since these phases are observed for densities higher than $\rho=0.1 \mu \mathrm{m}^{-2}$, i.e., when the closely moving colloids are forced to interact. For dilute systems [see Fig. 2(a)], the dynamics is similar to Ref. [19], and can be separated in two rather than three regimes before the pinned state. In particular, when single particles desynchronize with the ratchet, their motion become intermittent, alternating running with oscillating phases. For dense systems $\left[\rho>0.1 \mu \mathrm{m}^{-2}\right.$, see the inset of Fig. 2(a)], the average speed drops by a factor 6 due to the presence of traffic jams. In contrast, in regime III, $\left\langle v_{y}(t)\right\rangle$ slightly increases with $\rho$ since the mobile particles increase their speed in order to circumvent the pinned domains.

In regime II, the effect of the traffic jams on the dynamic organization of the system emerges upon measuring the evolution of the local density field. Figure 3(a) shows the spatiotemporal plot of the density field $\rho(y, t)$ averaged along the $x$ direction. The graph reveals that the jams do not disappear with time, but generate large scale fluctuations in the form of density waves [see Fig. 3(b)]. These waves propagate forward relative to the direction of motion and with an average speed $\left\langle v_{w}\right\rangle\left\langle\left\langle v_{y}\right\rangle\right.$ [see Fig. 3(c)], thus backward relative to the individual particles, extending up to $\sim 100 \mu \mathrm{m}$ in length. In this regime, the collective dynamics is closer to granular flow [20] or microfluidic droplets [21] than vortex matter. In regime III, the presence of the pinned domains hinders the formation of such waves, and the density field is almost constant with time (see Fig. 2 in the Supplemental Material [13]).

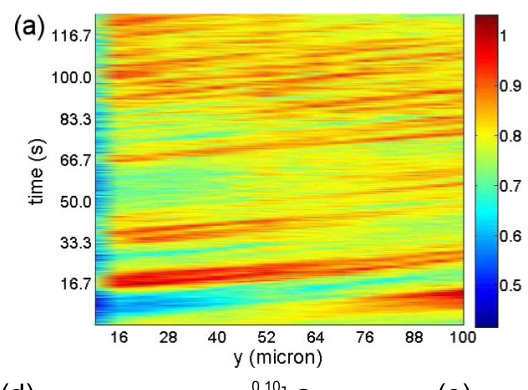

(b) 1.0

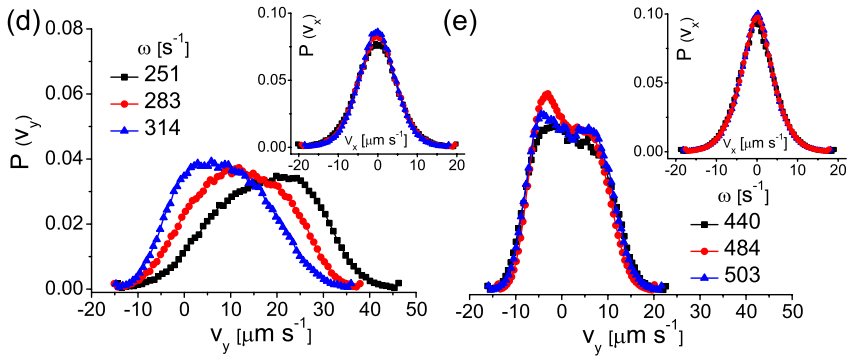

FIG. 3 (color online). (a) Space-time diagram of the normalized density field $\rho(y, t) / \rho_{0}$ with $\rho_{0}=0.15 \mu \mathrm{m}^{-2}$ in regime II $\left(\omega=251 \mathrm{~s}^{-1}\right)$. (b) $\rho(y, t) / \rho_{0}$ versus position $y$ at two different times. (c) Averaged velocity of the density waves $\left\langle v_{w}\right\rangle$ normalized with respect to $\left\langle v_{y}\right\rangle$ versus frequency in regime II. (d),(e) Normalized velocity distributions $P\left(v_{y}\right)$ in the driving direction for regime II (d), and regime III (e). Inset shows the corresponding PDFs along the transversal direction.

Another way to distinguish regimes II and III consists in measuring the probability distribution function (PDF) of the velocity $P(v)$, as shown in Figs. 3(d) and 3(e). For disordered flow, the PDF is broad, and the positions of the central peaks decrease as the driving increases. In the two phase flow, instead, $P\left(v_{y}\right)$ becomes bimodal, with a zero velocity peak, related to the pinned domains, and a second peak, related to the average speed of the flowing particles. This type of structure of the PDF is again reminiscent of plastic flow for vortices in dirty superconductors [22]. Increasing the frequency shifts the second peak towards the first one, and finally, in regime IV, both peaks merge into a Gaussian PDF centered on zero. In both regimes, the PDFs in the transversal direction are well fitted by Gaussian functions [see the inset of Figs 3(d) and 3(e)].

In contrast to previous works with driven charged colloids, this system allows us to easily tune in real time the particle interactions by varying the ellipticity of the applied field. This feature is demonstrated in Fig. 4, which shows two images of particles subjected to an elliptically polarized field with frequency $\omega=314 \mathrm{~s}^{-1}$ (regime II) and a ratio between the amplitudes $H_{z} / H_{y}=0.27$ (a) and $H_{z} / H_{y}=3.6$ (b) (see the Supplemental Material for movies [13]). When $H_{z} / H_{y}<1$, the particles assemble into traveling chains along the $y$ direction, due to attractive dipolar interactions. The average length and speed of the chains increase as $H_{z} / H_{y}$ decreases [see Fig. 4(c)]. On the other hand, when $H_{z} / H_{y}>1$, the particles repel each 

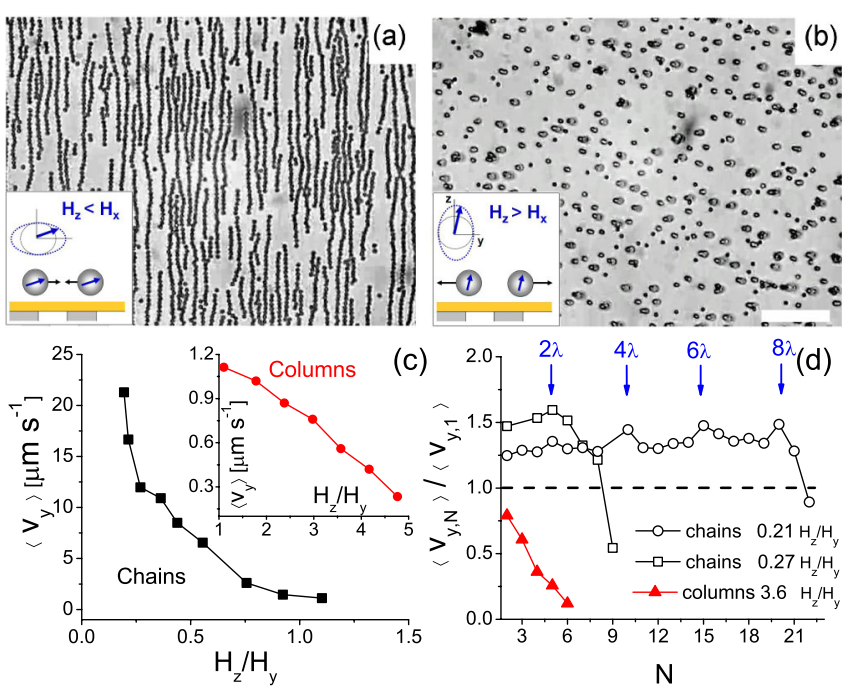

FIG. 4 (color online). (a),(b) Microscope images of paramagnetic colloids subjected to an elliptic rotating magnetic field with frequency $\omega=314 \mathrm{~s}^{-1}$ and ratio between components $H_{z} / H_{y}=0.27$ (a) and $H_{z} / H_{y}=3.6$ (b). (c) Average velocity $\left\langle v_{y}\right\rangle$ versus $H_{z} / H_{y}$ for chains, inset shows $\left\langle v_{y}\right\rangle$ for columns. (d) Average velocity of chains (empty squares and circles) and columns (filled triangles) $\left\langle v_{y, N}\right\rangle$ composed of $N$ particles, versus $N .\left\langle v_{y, 1}\right\rangle$ denotes the average velocity of one particle under the same conditions.

other, and when fast moving colloids approach slower ones, they assemble into columns, composed of 2-6 particles and with an average speed which decreases as the anisotropy of the field increases [see Fig. 4(c)]. Figure 4(d) shows that while the columns are the slower aggregates, the chains move faster, showing an increase in the rectification speed. This enhanced transport effect, which can increase the speed up to 1.6 times the speed of individual particles, is due to matching between the chain length and the spatial length scale of the substrate. Enhanced rectification in a string of driven attractive particles has been predicted in recent numerical works [23].

The dominant interactions between the moving particles are magnetic dipolar interactions. The pair-potential between two dipoles $\boldsymbol{m}_{i}, \boldsymbol{m}_{j}$ separated by $\boldsymbol{r}_{i j}$ is given by $U_{d d}=\frac{\mu}{4 \pi}\left(\frac{\boldsymbol{m}_{i} \boldsymbol{m}_{j}}{\boldsymbol{r}_{i j}^{3}}-\frac{3\left(\boldsymbol{m}_{i} \cdot \boldsymbol{r}_{i j}\right)\left(\boldsymbol{m}_{j} \cdot \boldsymbol{r}_{i j}\right)}{\boldsymbol{r}_{i j}^{5}}\right)$, and is maximally attractive (repulsive) for particles with moment parallel (normal) to $\boldsymbol{r}_{i j}$. In the magnetic ratchet, the dipoles try to follow the rotations of the field in the $(y, z)$ plane, and thus particles lying on the same BW are always repulsive, while particles located on two consecutive BWs can attract (or repel), depending on whether the field orientation $\vartheta$ (with respect to $y$ ) lies in the cones $\vartheta \in\left[n \pi-\vartheta_{m}, n \pi+\vartheta_{m}\right]$ (or $\left.\left[n \pi+\vartheta_{m}, \pi(n+1)-\vartheta_{m}\right]\right)$, with $n=0,1,2 \ldots$ and $\vartheta_{m}=54.7^{\circ}$ the "magic" angle [24], separating attractive from repulsive interactions. For circular ac fields, the particles experience no net interactions in the driving direction, since attraction and repulsion are equally balanced in time. Increasing the $H_{y}\left(H_{z}\right)$ component of the field and keeping the other fixed increases the time of attraction (or repulsion) between the particles during a field period, inducing a net interaction when averaged over many field cycles.

To conclude, we observe a rich nonequilibrium behavior in a monolayer of ratcheting paramagnetic colloids. Although the experiments are based on the manipulation of a structured magnetic substrate (the FGF), the underlying physics of the observed phenomena is common to many other driven systems characterized by collective transport in pinning potentials. It also unveils new perspectives in the use of a colloidal model system to study dynamics and cooperative motion with tunable interactions. In particular, an immediate extension of this work includes the use of bidisperse particles to study sorting during transport and the use of functionalized particles to move collectively large biological cargos.

I acknowledge Tom $\mathrm{H}$. Johansen for providing the garnet film, M. C. Miguel and T. M. Fischer for stimulating discussions, and J. Ortín for laboratory support. This work was supported by the programs "Ramon y Cajal" (RYC2011-07605), FIS2011-13771-E, and FIS2011-15948-E.

*ptierno@ub.edu

[1] H. J. Jensen, A. Brass, and A. J. Berlinsky, Phys. Rev. Lett. 60, 1676 (1988); N. Grønbech-Jensen, A. R. Bishop, and D. Domínguez, Phys. Rev. Lett. 76, 2985 (1996); C. J. Olson, C. Reichhardt, and F. Nori, Phys. Rev. Lett. 80, 2197 (1998).

[2] D.S. Fisher, Phys. Rev. B 31, 1396 (1985); S. Bhattacharya, J.P. Stokes, M. J. Higgins, and R. A. Klemm, Phys. Rev. Lett. 59, 1849 (1987); G. Grüner, Rev. Mod. Phys. 60, 1129 (1988).

[3] J.P. Hirth and J. Lothe, Theory of Dislocations (John Wiley \& Sons, New York, 1982); A. Carpio and L. L. Bonilla, Phys. Rev. Lett. 90, 135502 (2003).

[4] O. Narayan and D.S. Fisher, Phys. Rev. B 48, 7030 (1993); M. Dong, M. C. Marchetti, A. A. Middleton, and V. Vinokur, Phys. Rev. Lett. 70, 662 (1993); P. Chauve, P. Le Doussal, and K. J. Wiese, Phys. Rev. Lett. 86, 1785 (2001); O. Duemmer and W. Krauth, Phys. Rev. E 71, 061601 (2005).

[5] R. Seshadri and R. M. Westervelt, Phys. Rev. Lett. 70, 234 (1993); J. Hu and R. M. Westervelt, Phys. Rev. B 51, 17279 (1995).

[6] F. I. B. Williams, P. A. Wright, R. G. Clark, E. Y. Andrei, G. Deville, D. C. Glattli, O. Probst, B. Etienne, C. Dorin, C. T. Foxon, and J. J. Harris, Phys. Rev. Lett. 66, 3285 (1991); X. Zhu, P. B. Littlewood, and A. J. Millis, Phys. Rev. B 50, 4600 (1994); C. Reichhardt, C. J. Olson, N. Grønbech-Jensen, and F. Nori, Phys. Rev. Lett. 86, 4354 (2001).

[7] O. Braun, M. Paliy, and B. Hu, Phys. Rev. Lett. 83, 5206 (1999); J. Tekic, O. M. Braun, and B. Hu, Phys. Rev. E 71, 026104 (2005). 
[8] A. Pertsinidis and X. S. Ling, Phys. Rev. Lett. 100, 028303 (2008).

[9] P. T. Korda, M. B. Taylor, and D. G. Grier, Phys. Rev. Lett. 89, 128301 (2002); K. Mangold, P. Leiderer, and C. Bechinger, Phys. Rev. Lett. 90, 158302 (2003).

[10] T. Bohlein, J. Mikhael, and C. Bechinger, Nature Mater. 11, 126 (2012).

[11] P. Tierno, F. Sagués, T.H. Johansen, and T. M. Fischer, Phys. Chem. Chem. Phys. 11, 9615 (2009).

[12] P. Tierno, S. V. Reddy, T. H. Johansen, and T. M. Fischer, Phys. Rev. E 75, 041404 (2007); P. Tierno, P. Reimann, T.H. Johansen, and F. Sagués, Phys. Rev. Lett. 105, 230602 (2010).

[13] See Supplemental Material at http://link.aps.org/ supplemental/10.1103/PhysRevLett.109.198304 for more experimental details, analysis of data and videos showing the particle dynamics.

[14] In a previous work [11] based on an oscillating field, it was found that above a critical frequency $\left(\omega \sim 100 \mathrm{~s}^{-1}\right)$, the overdamped particles were unable to follow the ratchet modulations, and their velocities vanished immediately. This was due to deep and stiff potential wells $\left(>100 k_{B} T\right)$, which hinder slippery motion between the BWs.

[15] The strength of the magnetic potential was determined by measuring the residence probability $p(y)$ of a dilute sample (negligible interactions) perpendicularly to the BWs lines, similar to Ref. [16]. At equilibrium (no external field), we apply the Boltzmann distribution, $p(y)=$ $p_{0} e^{\left(-U / k_{B} T\right)}$, and find the potential inverting this equation.
[16] C. Bechinger, M. Brunner, and P. Leiderer, Phys. Rev. Lett. 86, 930 (2001).

[17] C. Reichhardt and C. J. O. Reichhardt, Phys. Rev. E 72, 032401 (2005).

[18] As shown in Video 4 in the Supplemental Material [13], the pinned particles are in reality not immobile, but perform fast back and forth oscillations above the BWs, waiting to receive enough energy from other particles to synchronize back with the ratchet.

[19] B. B. Yellen, R. M. Erb, H. S. Son, R. Hewlin Jr., H. Shang, and G. U. Lee, Lab Chip 7, 1681 (2007).

[20] G.W. Baxter, R.P. Behringer, T. Fagert, and G. A. Johnson, Phys. Rev. Lett. 62, 2825 (1989); H.M. Jaeger, S.R. Nagel, and R.P. Behringer, Rev. Mod. Phys. 68, 1259 (1996); O. Moriyama, N. Kuroiwa, M. Matsushita, and H. Hayakawa, Phys. Rev. Lett. 80, 2833 (1998).

[21] T. Beatus, T. Tlusty, and R. Bar-Ziv, Phys. Rev. Lett. 103, 114502 (2009).

[22] M.C. Faleski, M.C. Marchetti, and A. A. Middleton, Phys. Rev. B 54, 12427 (1996).

[23] J. Dzubiella, G. P. Hoffmann, and H. Löwen, Phys. Rev. E 65, 021402 (2002); C. Reichhardt and C. J. Olson Reichhardt, Phys. Rev. E 74, 011403 (2006); A. Pototsky, A. J. Archer, M. Bestehorn, D. Merkt, S. Savelév, and F. Marchesoni, Phys. Rev. E 82, 030401 (2010).

[24] P. Tierno, R. Muruganathan, and T. M. Fischer, Phys. Rev. Lett. 98, 028301 (2007). 